\section{SAT0127 UNFAVORABLE BODY COMPOSITION ALREADY AT THE ONSET OF CLINICAL ARTHRITIS}

S.A. Turk ${ }^{1}$, D. van Schaardenburg ${ }^{1,2}$, M. Boers ${ }^{3}$, S. de Boer ${ }^{1}$, C. Fokker ${ }^{1}$, W.F. Lems ${ }^{1,3}$, M.T. Nurmohamed ${ }^{1,3},{ }^{1}$ Amsterdam Rheumatology and immunology Center| Reade; ${ }^{2}$ Amsterdam Rheumatology and immunology Center| Academic Medical Center; ${ }^{3}$ Amsterdam Rheumatology and immunology Center| VU University Medical Center, Amsterdam, Netherlands

Background: Rheumatoid arthritis is associated with an increased cardiovascular (CV) risk. There is mounting evidence that inflammation is involved in the pathogenesis thereof (1). An unfavorable body composition is, independently, associated with an increased CV risk, and often present in established arthritis patients. This unfavorable composition is a loss of muscle mass (sarcopenia), in the presence of a stable or even increased (abdominal) fat mass (sarcopenic obesity) (2).

Objectives: Currently it is unknown when this unfavorable body composition develops. Therefore, we compared body composition in DMARD-naïve early arthritis patients with non-arthritis controls and explored the association with disease activity and traditional $\mathrm{CV}$ risk factors.

Methods: A total of 317 consecutive early arthritis patients and 1268 age, gender and ethnicity matched non-arthritis controls (3) had a Dual-energy X-ray absorptiometry scan to assess lean (muscle) mass index (LMI), fat mass index (FMI), fat mass distribution (arms and legs versus trunk) and android to gynoid fat mass ratio. For the obesity definition, the cut offs of Gallaher et al. were applied. For the sarcopenia definition, cut offs based on the control group were applied (mean minus two times SD). In addition, a disease activity score, erythrocyte sedimentation rate, lipid profile (total cholesterol, HDL, LDL and triglycerides) and blood pressure assessments were done.

Results: Early arthritis patients (84\% fulfilling the 2010 ACR/EULAR criteria) had a significantly lower mean LMI $(p<0.01)$ compared with controls. Female patients had a higher mean FMI $(p<0.01)$ and more fat was distributed to the trunk in patients (females $p<0.01$, males $p=0.07$ ) (table). The prevalence of an unfavorable body composition (i.e. sarcopenia and sarcopenic obesity) was higher than in controls, for females $5.0 \%$ versus $1.3 \%$, OR: $4.2, p<0.01$ and for males $8.2 \%$ versus $1.5 \%$, OR: $5.7, \mathrm{p}<0.01$ (figure). A higher $\mathrm{FMI}$, more android fat and more fat distributed to the trunk were associated with higher blood pressure and lipid levels (and lower HDL levels). There was no clear relationship between body composition parameters and disease activity.

Table 1. Body composition of early arthritis patients and non-arthritis controls

\begin{tabular}{lcccc}
\hline & $\begin{array}{c}\text { Arthritis } \\
\text { females }\end{array}$ & $\begin{array}{c}\text { Control } \\
\text { females }\end{array}$ & $\begin{array}{c}\text { Arthritis } \\
\text { males }\end{array}$ & $\begin{array}{c}\text { Control } \\
\text { males }\end{array}$ \\
\hline Fat mass index (mean, SD) & $27.6(5.3)$ & $26.6(4.6)^{\star}$ & $26.8(4.4)$ & $27.4(3.6)$ \\
$\%$ of fat distributed to the trunk (mean, SD) & $52.2(6.3)$ & $49.4(6.5)^{\star}$ & $58.8(6.4)$ & $57.6(5.6)$ \\
Android to gynoid fat mass ratio (mean, SD) & $0.5(0.2)$ & $0.5(0.2)$ & $0.7(0.2)$ & $0.8(0.2)^{\star}$ \\
Lean mass index (mean, SD) & $6.6(0.9)$ & $6.7(0.8)^{\star}$ & $8.0(1.1)$ & $8.6(1.0)^{\star}$ \\
Obese (n,\%) & $99(45.2)$ & $334(38.4)$ & $50(52.1)$ & $222(57.7)$ \\
Sarcopenic (n,\%) & $11(5.0)$ & $11(1.3)^{\star}$ & $8(8.2)$ & $6(1.5)^{\star}$ \\
Sarcopenic obesity (n,\%) & $2(0.9)$ & $2(0.2)$ & $4(4.2)$ & $3(0.8)^{\star}$ \\
\hline
\end{tabular}

$\mathrm{n}=$ number, $\mathrm{SD}=$ standard deviation, $\%==$ percentages ${ }^{*} \mathrm{p}$-value $<0.05$ between arthritis and non-arthritis controls.

Figure. Prevalence of sarcopenia and sarcopenic obesity in early arthritis patients compared with non-arthritis controls.

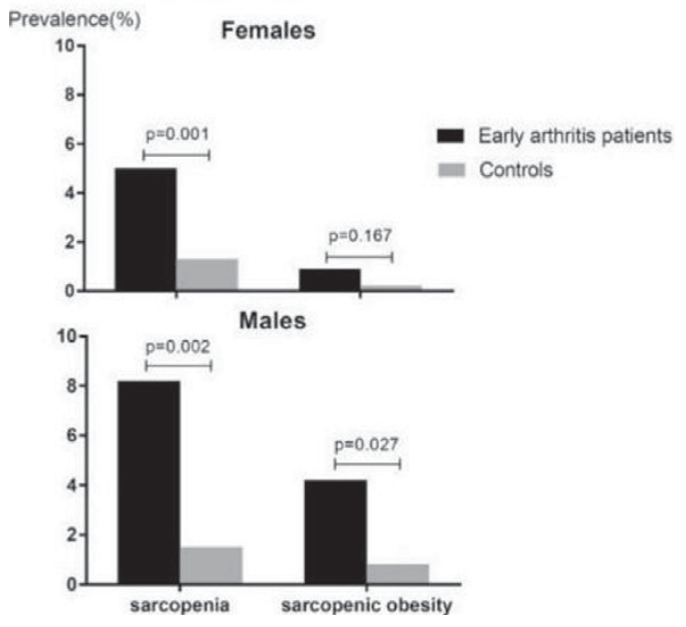

Conclusions: Patients at the clinical onset of arthritis more often have an unfavorable body composition (sarcopenia and sarcopenic obesity) than nonarthritis controls. An unfavorable body composition was associated with higher blood pressure and lipid levels (and lower HDL levels).

References:

[1] Curr Vasc Pharmacol 2010; 8(2):285-292.

[2] Age (Dordr) 2015; 37(6):121.

[3] Eur J Epidemiol 2015; 30(8):661-708
Disclosure of Interest: None declared

DOI: 10.1136/annrheumdis-2017-eular.2174

\section{SAT0128 CLINICAL FEATURES AND EVOLUTION OF PULMONARY FUNCTION IN A SINGLE-CENTER COHORT OF PATIENTS WITH RHEUMATOID ARTHRITIS RELATED INTERSTITIAL LUNG DISEASE}

S.C. Rodriguez-García, R.A. Castellanos-Moreira, M.V. Hernandez-Miguel, J. Ramirez, A. Cuervo, J. Cañete, R. Sanmarti-Sala. Rheumatology, Hospital Clinic Barcelona, Barcelona, Spain

Background: Interstitial lung disease (ILD) is an extra-articular manifestation of Rheumatoid Arthritis (RA), has been related with a poor prognosis. However, there is a lack of clinical data regarding its evolution with different treatment approaches.

Objectives: Our aim was to assess clinical features and evolution of ILD in a cohort of RA patients from a tertiary hospital

Methods: Single-centre retrospective observational study including all patients diagnosed with ILD with a previous or posterior diagnosis of RA evaluated from January 2007 to December 2016 in the Rheumatology Department of a university hospital.

Variables analysed: age, sex, smoking status, duration of RA, presence and titers of ACPA (antiCCP2) and rheumatoid factor (RF), presence of erosions, use of glucocorticoids (GC), synthetic and biological DMARDs before and after ILD diagnosis and presence of other extra-articular manifestations.

We also analysed: duration of ILD, HRCT (High Resolution Computed Tomography) patterns and pulmonary function tests (PFT) at baseline and during follow-up to assess the evolution of the disease. The PFT results were evaluated according to the American Thoracic Society/European Respiratory Society definitions (1). Results: Twenty-one patients (18 women) were included, mean age $68.4 \pm 11.9$, mean RA duration $12.3 \pm 8.2$ years. $9.5 \%$ were current smokers and $33.3 \%$ former smokers. $61.9 \%$ were ACPA + $52.4 \%$ with high basal titers, median ACPA titers: $698)$ and $81 \% \mathrm{RF}+(47.6 \%$ with high basal titers). $71.4 \%$ had erosions and 5 patients $(23.8 \%)$ had extra-articular manifestations, mainly rheumatoid nodules.

RA diagnosis was made after that of ILD in 4 patients; mean elapsed time $1.56 \pm 1.26$ years. In the other 17 patients, the mean duration of RA until ILD diagnosis was $11.2 \pm 7.2$ years.

Currently 17 subjects (85\%) receive GC; 4 (19\%) in monotherapy and 11 (57.9\%) synthetic DMARDs (5 methotrexate, 3 leflunomide (LEF), 1 hydroxychloroquine (HCQ) and 2 with double therapy (HCQ/AZA and HCQ/LEF)).

Four patients are currently treated with biological therapy (2 abatacept (ABA), 1 rituximab and 1 etanercept (ETN)), all of them in monotherapy.

Five patients received TNFi prior to ILD diagnosis (3 ETN, 1 infliximab and 1 adalimumab). Biological therapy was withdrawn in 3 cases and switched to ABA in the two remaining ones.

The median duration of EPID was 2.5 (range $0.7-11.9$ ) years. HRCT patterns were: non-specific interstitial pneumonia (NSIP) $52.9 \%$, usual interstitial pneumonia (UIP) $17.6 \%$, cryptogenic organized pneumonia (COP) $11.8 \%$, and other patterns $17.7 \%$.

14 patients had baseline and follow-up PFT: 9 (64.3\%) remained stable; 3 (21.4\%) improved and $2(14.3 \%)$ worsened ( 1 had received previous ETN). There was a trend for worsening of PFT in patients with UIP pattern.

No patient died from ILD during this period

Conclusions: In our RA-ILD cohort, NSIP was the most common HRCT pattern and PFT remained stable in most patients during follow-up. Five patients $(23.8 \%)$ had received TNFi prior to ILD diagnosis and only one of them showed worsening of PFT during ETN treatment, with clinical improvement after withdrawal.

References:

[1] Pellegrino R et al. Eur Respir J. 2005;26:94868.

Disclosure of Interest: None declared

DOI: 10.1136/annrheumdis-2017-eular.3284

\section{SAT0129 EXPLORATION OF COMORBIDITY INDICES IN AN ETHNIC RHEUMATOID ARTHRITIS SUBSET}

S. Dowell ${ }^{1}$, G. Kerr ${ }^{2}$, C. Swearingen ${ }^{3}$, M. Quinones ${ }^{1}$, J. Berrian ${ }^{1}$, S. Hochberg ${ }^{1}$ on behalf of EMRAC Working Group. ${ }^{1}$ Rheumatology, Howard University Hospital; ${ }^{2}$ Rheumatology, Washington VAMC, Washington; ${ }^{3} \mathrm{NYU}$, New York, United States

Background: Comorbidity is common in ethnic patients with rheumatoid arthritis (RA) and often impact choice of DMARD therapy and clinical outcomes. In addition to simple summation of comorbidities, composite indices have been proffered as better predictors of poor clinical outcomes in patients with rheumatoid arthritis ${ }^{1}$. However, their applicability in ethnic RA subsets is unknown.

Objectives: To compare composite to mean comorbidity indices in ethnic subsets with rheumatoid arthritis.

Methods: Patients enrolled in the Ethnic Minority RA Consortium (EMRAC), with at least 6 months of followup data were analyzed. At enrollment, sociodemographic data and RA disease status, rheumatic disease comorbidity index (RCDI) ${ }^{1}$ and comorbidity count (COUNT) ${ }^{1}$ were analyzed amongst ethnic subsets. Spearman correlation was estimated between RCDI and COUNT while Poisson regression 